\title{
Test of a model for number-averaging behavior'
}

\author{
Norman H. Anderson \\ UNIVERSITY OF CALIFORNIA, LOS ANGELES
}

\begin{abstract}
Abstraet
Ss performed fairly well at sequential number-averaging, but showed a definite recency effect. A model in which the response is a weighted sum of previous stimuli was supported, and an orderly serial position curve was obtained.
\end{abstract}

\section{Problem}

Ss saw a sequence of 2-digit numbers, and estimated the average of the sequence after each successive number. Experiment 1 used 2 sequences chosen to exhibit some gross features of the behavior. Experiment 2 was designed to (a) test the hypothesis that the response was a weighted sum of the numbers seen, and (b) to measure the influence of each preceding serial position on the present response.

\section{Experiment 1}

\section{Method}

Eight cards, each listing a 2-digit number, were shown singly for approximately $7 \mathrm{sec}$. each. On seeing each successive card, $\mathrm{S}$ estimated the average of all the numbers in the sequence. $\mathrm{S}$ was instructed to base each estimate on the accumulated information, and not to worry unduly about mathematical correctness but to give "what your feeling about the average is."

After a practice sequence, 4 test sequences were given in latin square order. Sequences IA and IIA are given in Fig. 1. Sequences IB and IIB were respectively 48 and 26 greater at each position. The data for A and B sequences were pooled. Twenty males from introductory psychology were run individually.

\section{Results}

Figure 1 shows (a) the actual number stimuli; (b) the arithmetic cumulative means of these stimuli; and (c) the mean response at each serial position. The nearequality of the latter 2 curves indicates that Ss are fairly accurate for these particular sequences. There is, however, a definite recency effect. At the last 2 positions of Sequence I, the change in response is greater than the change in number-mean, and this same recency is seen at every position in Sequence II.

\section{Experiment 2}

\section{Method}

Each $\mathrm{S}$ judged 3 practice and 8 test sequences, all of 7 2-digit numbers. A 15-sec. intersequence interval was spent rating cartoons. Ss were 30 males. Other details were as above.

The last number in each sequence was only a filler. The first 6 numbers were obtained as follows. At each serial position, 1 of a pair of numbers occurred. These 6 pairs were $(73,56),(55,38),(62,45),(44,27),(66$, $49)$, and $(51,34)$. In each pair the Hi and Lo numbers differ by 17 . With 2 numbers at each position, there are 64 possible sequences. This $2^{6}$ design was run in $1 / 8$ replication (Cochran \& Cox, 1957; p. 278). These 8 sequences allow estimation of the 6 main effects, with 1 residual df for interaction to test the weighted sum model. Number-pairs were balanced over Serial Position with a $6 \times 6$ latin square; this separates effect of Serial Position per se from possible effects of particular Number-pairs.

\section{Results}

Only the response at Serial Position 6 is considered. That response depends on the numbers at the first 6 serial positions of the sequence. The influence of each

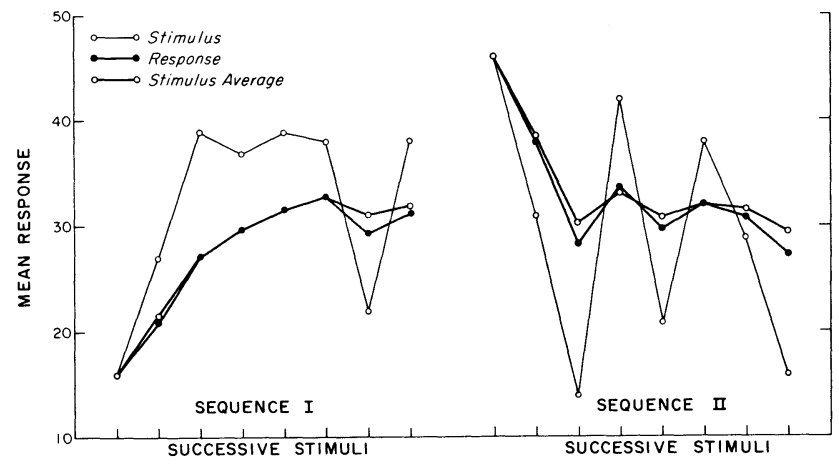

Fig. 1. Mean response at successive serial positions, Exp. 1. Faint line plots actual number stimuli for each Sequence. Paired heavy lines compare the response with the (cumulated) stimulus mean. 
serial position on that response is given in the first line of Table 1. The entry for Serial Position $\mathrm{k}$ is a difference of 2 means: the mean response for those sequences in which the kth number was Hi (e. g., 73), minus the mean response for those sequences in which the kth number was Lo (e. g., 56). The difference is thus the differential effect of the 2 numbers that could occur at Position k.

The entry of 5.25 for Serial Position 6 represents the influence of the number that is visibly present. This entry is by far the largest and reflects the recency observed in Experiment 1. The serial position curve then declines, at first rapidly, but only very gradually at the more remote positions where the entries are nearly equal and still considerably greater than 0 .

The main theoretical question concerns the validity of the weighted sum model. As noted in the preceding paper (Anderson, 1964), the interaction term of the design provides the test. The mean interaction, computed as a specific comparison, was -.13 which, with a standard error of .24 , is not significant. This result, and the neatness of the serial position curve, support the model.

Each $\mathrm{S}$ received a specific comparison score for each of the 6 main effects of the design, the means of which are in fact just the entries in the first line of Table 1. Their separate standard errors, in the second line, show that each is significantly greater than 0 . In the full repeated measurements analysis, Serial Position was significant, $F(5,120)=9.99$, but neither Number-pairs, their Order of Presentation, nor the Residual approached significance.

The above interpretation of Table 1 entries as serial position effects requires a brief justification. Nonsig-

Table 1. Effect of Preceding Serial Positions on Response at Serial Position 6

$$
\text { Serial Position }
$$

$\begin{array}{lcccccc} & 1 & 2 & 3 & 4 & 5 & 6 \\ & & & & & & \\ \text { Mean } & 2.03 & 2.07 & 2.12 & 2.32 & 3.02 & 5.25 \\ \text { Standard error } & .44 & .27 & .33 & .41 & .33 & .40\end{array}$

nificance of the interaction indicates (tentatively) that the model is correct. If the model is correct, then the Position $\mathrm{k}$ entry equals $\mathrm{w}_{\mathrm{k}}(\mathrm{Hi}-\mathrm{Lo})$, where $\mathrm{w}_{\mathrm{k}}$ is the weight associated with Position $\mathrm{k}$, and $\mathrm{Hi}$ - Lo is the effective difference between the $\mathrm{Hi}$ and Lo numbers at Position k. All Hi - Lo differences were equal numerically, and the nonsignificance of Number-pairs indicates that they were psychologically equal as well. It is thus concluded that the Table 1 entries are proportional to the $\mathrm{w}_{\mathrm{k}}$ values, and hence interpretable as serial position effects per se.

\section{Discussion}

The number-averaging task has intrinsic interest, and the behavior seems orderly enough to warrant further work. In particular, the results suggest that the serial position effects are relatively free from the influence of other factors, such as sequence patterning.

Number-averaging may be considered as sequential information integration. Another such task is impression formation in which $\mathrm{S}$ receives a sequence of personalitytrait adjectives and rates his liking of the person so described. Assuming that each adjective has a certain value for $S$, it may be conjectured that $S$ is there also engaged in sequential number-averaging, and there is some evidence to support this view (Anderson, in press).

The theoretical analysis that has been illustrated here may be applicable more generally in the study of sequential information integration. Moreover, the simple number-averaging data may provide a useful baseline for the interpretation of behavior with more complex verbal stimuli.

\section{References}

ANDERSON, N. H. Note on weighted sum and linear operator models. Psychon. Sci., 1964, 1, 189-190. ANDERSON, N. H. Primacy effects in personality impression formation using a generalized order effect paradigm. J. abnorm. soc. Psychol., in press.

COCHRAN, W. G., \& COX, G. M. Experimental designs. New York: Wiley, 1957.

\section{Note}

1. This work was supported by NSF Grant GB-1470. I wish to thank Ann Norman for her assistance. 